\title{
Diet and asthma in Dutch school children (ISAAC-2)
}

\author{
C Tabak, A H Wiiga, G de Meer, N A H Janssen, B Brunekreef, H A Smit

Background: The rise in the prevalence of asthma in western societies may be related to changed dietary habits. Epidemiological studies in children have shown inverse associations of asthma related outcomes with intake of fruits, vegetables, dairy and whole grain products, and fish. In contrast to most previous studies, we used both questionnaire and clinical data to define asthma.

See end of article for authors' affiliations ......................

Correspondence to: Dr H A Smit, Center for Prevention and Health Services Research (PZO)

National Institute of Public Health and the Environment, P O Box 1 , 3720 BA Bilthoven, The Netherlands; jet.smit@ rivm.nl

Received 7 March 2005 Accepted 10 October 2005 Published Online First 21 October 2005
Methods: Intake of the abovementioned foods was studied in relation to asthma in 598 Dutch children aged 8-13 years. Dietary intake was estimated using a parent completed semi-quantitative food frequency questionnaire. Current wheeze and current asthma were defined based on questionnaire data. More complex end points were defined using information on bronchial hyperresponsiveness (BHR) and atopic sensitisation as well. Linear associations were studied using logistic regression analysis and odds ratios presented for the highest versus the lowest tertile of intake. In the final models, adjustments were made for maternal educational level, foreign descent, and total energy intake.

Results: The intake of whole grain products and of fish was inversely associated with asthma. Adjusted odds ratios for the independent associations with whole grains and fish were 0.46 (95\% $\mathrm{Cl} 0.19$ to 1.10 ) and $0.34(95 \% \mathrm{Cl} 0.13$ to 0.85$)$ for current asthma and $0.28(95 \% \mathrm{Cl} 0.08$ to 0.99$)$ and $0.12(95 \% \mathrm{Cl}$ 0.02 to 0.66 ) for atopic asthma with BHR. Similar results were observed for current wheeze and atopic wheeze with BHR. Intake of (citrus) fruits, vegetables, and dairy products showed no clear associations with asthma end points.

Conclusions: Our findings suggest that a high intake of whole grain products and fish may have a protective effect against asthma in children. t has been postulated that the rise in the prevalence of asthma in western societies may be related to changed dietary habits since the 1950s-specifically, decreased intake of fruits, vegetables and fish. ${ }^{1}$ Another factor that may be important is that, as a result of recommendations to substitute saturated fatty acids with n- 6 fatty acids to reduce serum cholesterol concentrations, the current western diet is rich in $\mathrm{n}-6$ fatty acids. ${ }^{2}$ The biological mechanisms by which these dietary changes may affect asthma all seem to include airway inflammation.

Antioxidants are thought to reduce airway inflammation by protecting the airways against oxidants from both endogenous (activated inflammatory cells) and exogenous (for example, cigarette smoke) sources. ${ }^{3}$ Fruits, vegetables, and whole grain products are rich in antioxidant vitamins (vitamin $\mathrm{C}$, vitamin E, $\beta$-carotene) and other substances with antioxidant capacity (including phenolic acids and phytic acid in whole grains ${ }^{4}$ ). Fish oils are rich sources of n-3 fatty acids. Both n-6 and n-3 fatty acids are precursors for the production of pro-inflammatory mediators called eicosanoids, including prostaglandins and leukotrienes. Increasing the intake of $n-3$ fatty acids shifts the balance towards eicosanoids derived from n-3 fatty acids, which are thought to be biologically less active. ${ }^{2}$ As a result, the present western diet may promote airway inflammation, a major characteristic of asthma.

Epidemiological studies in children have shown inverse associations with asthma related outcomes for (citrus) fruits, ${ }^{6-8}$ vegetables, ${ }^{8-11}$ whole grain products, ${ }^{12}$ and fish. ${ }^{613-16}$ Furthermore, inverse associations with asthma have also been observed in children for butter ${ }^{13}{ }^{17}$ and (whole or farm) milk..$^{10} 12{ }^{19} \mathrm{~A}$ high intake of the latter products, being rich in saturated fatty acids, may reduce the relative contribution of unsaturated fatty acids, including n-6 fatty acids, to total fat intake.

We studied the intake of fruits, vegetables, dairy and whole grain products, and fish in relation to asthma in 598 Dutch children aged 8-13 years, also considering independent and combined effects of foods associated with asthma. The main asthma end points studied were current wheeze and current asthma based on questionnaire data. Information from tests on bronchial hyperresponsiveness (BHR) and of sensitisation to common allergens (skin prick testing) available for these children allowed us to define more complex asthma end points based on both questionnaire and clinical data (atopic wheeze with BHR and atopic asthma with BHR).

\section{METHODS}

\section{Study population}

As part of the International Study on Allergy and Asthma in Childhood 2 (ISAAC-2), during the period 1997-8 the effect of living close to freeways on respiratory health was studied in Dutch children aged $7-13$ years..$^{20}$ Parents of all children were asked to complete the ISAAC questionnaire on respiratory and allergic symptoms, completed with questions on pets and passive smoking. Children aged $8-13$ years were invited to participate in tests of BHR and of sensitisation to common allergens (including skin prick testing). In addition, a dietary questionnaire was sent to the parents of all children who participated in the BHR testing.

Adequate dietary information was collected from 812 children aged 8-13 years. Information on atopic sensitisation from skin prick tests and on BHR, as well as questionnaire data used to define asthma end points (see data analysis), was available for 609 of these children. An additional 11 children had missing values on one or more potential confounders considered in the final analysis. The final study population therefore consisted of 598 children.

Abbreviations: BHR, bronchial hyperresponsiveness; $\mathrm{FEV}_{1}$, forced expiratory volume in 1 second; $F F Q$, food frequency questionnaire 
Medical ethical approval was obtained from the ethical board of Wageningen University, the Netherlands. Parents gave written informed consent for each test separately.

\section{Diet}

Dietary intake was estimated using a semi-quantitative food frequency questionnaire (FFQ) developed and validated for the Dutch EPIC (European Prospective Investigation into Cancer and Nutrition) cohort of adults. ${ }^{21}$ The EPIC FFQ contained questions on the absolute frequency of consumption of 79 main food items (possible answers: times per day/ week/month/year or never) and, for several food items, additional questions on the relative consumption frequency of sub-items (possible answers: always or mostly/often/ sometimes/seldom or never). For 21 main foods the questionnaire contained colour photographs of 2-4 differently sized portions (possible answers: one of the amounts shown, less than the smallest, or more than the largest amount shown). From the information obtained, the habitual consumption of 178 food items during the previous year could be estimated. Energy intake was calculated using the 1996 Dutch food composition table. ${ }^{22}$

Parents were asked to fill in the EPIC FFQ for their child participating in ISAAC-2. Standard procedures for cleaning and processing of the data, as developed for the Dutch adult cohort, were evaluated for use in children. Since in children portion sizes may depend on both sex and age, we imputed not the sex-specific mean value but the age-specific $(8-9,10-$ $11,12-13$ years) and sex-specific mean value of the respondents if the portion size of a food was missing ( $\mathrm{n}=0-39$ of 598 for different foods). If a cross instead of a number was placed in one of the four boxes (per day/week/ month/year) of questions into absolute food consumption frequencies, we imputed the average number responded into that box. If this average was based on less than five children, the lowest possible number $(=1)$ was imputed.

Fruit intake did not include consumption of fruit juices. Vegetable intake did not include consumption of potatoes, legumes, and vegetable juices. Intake of "dairy other than low fat" was defined as intake of dairy products with a total fat content of $1.5 \mathrm{~g}$ or more per $100 \mathrm{~g}$ product, and intake of whole grain products as intake of wholemeal bread and of unrefined grains (for example, brown rice).

\section{Asthma}

The ISAAC questionnaire provided information on respiratory symptoms, current use of asthma medication, and a large range of potential confounders. ${ }^{23}$ Current wheeze was defined as wheeze in the past 12 months and ever asthma as a positive response to the question "Did your child ever have asthma?" Children were defined as having current asthma if they fulfilled at least one of the following three criteria: (1) ever asthma in combination with current wheeze; (2) ever asthma in combination with current use of a bronchodilator ( $\beta$ agonists or anticholinergics); (3) current use of specific asthma medication (corticosteroids, cromoglycate, nedocromil, or xanthines). Atopic wheeze with BHR was defined as current wheeze in combination with both atopy and BHR. Atopic asthma with BHR was defined as current asthma in combination with both atopy and BHR.

BHR tests were performed according to the ISAAC protocol. $^{24} 25$ Children with a baseline forced expiratory volume in 1 second $\left(\mathrm{FEV}_{1}\right)$ of $<75 \%$ predicted were excluded from BHR testing. Parents were asked to stop their children's use of salbutamol 6 hours before the test and that of antihistamines 48 hours before the test. The challenge protocol consisted of inhalation of a hypertonic (4.5\%) saline aerosol for $0.5,1,2,4$, and 8 minutes. After each inhalation step, two reproducible measurements of $\mathrm{FEV}_{1}$ were achieved of which the higher was selected. The test was stopped after completing all inhalation steps or when a fall in $\mathrm{FEV}_{1}$ of more than $15 \%$ of baseline was observed. A positive test (BHR) was defined as a fall in $\mathrm{FEV}_{1}$ of $\geqslant 15 \%$ after inhalation of maximal $23 \mathrm{ml}$ hypertonic saline. All lung function tests were performed according to ERS guidelines. ${ }^{26}$

Skin prick tests for atopic sensitisation were performed according to the standardised ISAAC-2 protocol. ${ }^{27}$ Standardised allergen extracts and controls were provided by the ALK Company (Horsholm, Denmark). Allergens tested were Dermatophagoides farinae, D pteronyssinus, cat, dog, Alternaria tenuis, mixed tree pollen, and mixed grass pollen. The test was performed on the volar side of the left forearm. A mean weal diameter of $3 \mathrm{~mm}$ or more was regarded as a positive reaction. ${ }^{28}$

\section{Data analysis}

All statistical analyses were performed using the SAS statistical package version 8.02 (Cary, USA). To estimate the (adjusted) prevalence of the studied asthma end points for levels of categorised dietary variables, PROC GLM (option LSMEANS) was used with the asthma outcome (yes/no) as independent variable. Since this method may give invalid SE values at low estimated prevalences, logistic regression analysis was used to test for differences between categories (using dummy variables).

Logistic regression analysis was used to study linear associations between dietary variables and asthma end points. The odds ratios (ORs) presented were calculated by multiplying the estimated regression coefficient $(\beta)$ by the difference between the mean intake of the studied food in the third versus the first tertile of intake (DIFF in either $\mathrm{g}$ /day or natural $\left.\log (\mathrm{g} / \mathrm{day}): \mathrm{OR}=\exp \left(\beta^{\star} \mathrm{DIFF}\right)\right)$. To evaluate the independent effects of foods associated with asthma, the dietary variables studied were entered simultaneously as independent variables in a single logistic regression model. To study the combined effect of whole grain products and fish, the prevalences of current wheeze and current asthma were compared between children with an intake of both foods in

Table 1 Characteristics of study population (ISAAC-2: $n=598$ )

\begin{tabular}{|c|c|}
\hline Variable & Mean (SD) \\
\hline $\begin{array}{l}\text { Age (years) } \\
\text { Height }(\mathrm{m}) \\
\text { Weight }(\mathrm{kg}) \\
\text { Total energy intake (MJ/day) }\end{array}$ & $\begin{array}{l}10.4(1.2) \\
1.48(0.09) \\
39.5(8.3) \\
9.4(2.2)\end{array}$ \\
\hline $\begin{array}{l}\text { Total fruits (g/day) } \\
\text { Citrus fruits (g/day) } \\
\text { Total vegetables (g/day) } \\
\text { Total dairy products (g/day) } \\
\text { Dairy other than low fat (g/day) } \\
\text { Total whole grain products (g/day) } \\
\text { Total fish (g/day) }\end{array}$ & $\begin{array}{l}171(108) \\
43(37) \\
94(42) \\
644(301) \\
470(253) \\
45(59) \\
7(7)\end{array}$ \\
\hline Variable & $\%$ \\
\hline $\begin{array}{l}\text { Current wheeze (yes) } \\
\text { Current asthma (yes) } \\
\text { Atopy (yes) } \\
\text { BHR (yes) } \\
\text { Sex (boys) } \\
\text { Educational level of mother (low*) } \\
\text { Foreign descent† (yes) } \\
\text { Ever asthma: parents } \ddagger \text { (yes) } \\
\text { Ever asthma: siblings } \uparrow \text { (yes) }\end{array}$ & $\begin{array}{r}8.9 \\
6.5 \\
29.4 \\
21.9 \\
52.7 \\
41.6 \\
16.1 \\
13.2 \\
15.2\end{array}$ \\
\hline \multicolumn{2}{|c|}{$\begin{array}{l}\text { *Intermediate secondary education or less. } \\
\text { †Child and/or one of the parents not born in the } \\
\text { Netherlands. } \\
\mp n=552 \text {. } \\
\text { In }=500 \text {. }\end{array}$} \\
\hline
\end{tabular}


the highest food specific tertile and children with an intake of both foods in the lowest food specific tertile.

In the final analyses, adjustments were made for educational level of the mother, foreign descent, and total energy intake. Preliminary analyses showed no confounding effects of age, sex, number of siblings, ever asthma in siblings, ever asthma in parents, smoking of the mother during pregnancy, being breastfed, passive smoking, the presence of pets in the home, or body mass index. $\mathrm{p}$ values $<0.05$ were considered statistically significant (two sided tests).

\section{RESULTS}

The characteristics of the study population $(n=598)$ are shown in table 1. The mean age of the children included in the study was 10 years and the children were almost equally distributed according to sex. Of the 53 children with current wheeze (prevalence 8.9\%), 37 were atopic and both atopy and bronchial hyperreactivity were observed in 24 . In the 39 children with current asthma (prevalence 6.5\%), 30 were atopic and both atopy and bronchial hyperreactivity were observed in 19. The mean daily intake was relatively high for fruits $(17 \mathrm{lg})$ and dairy products $(644 \mathrm{~g})$, while fish intake was low with a mean intake of only $7 \mathrm{~g}$ daily (table 1 ).

Table 2 shows the mean intake and crude prevalence of current wheeze and current asthma for tertiles of intake of the studied foods. A high intake of whole grain products and of fish was inversely associated with the prevalence of both outcomes. The observed prevalences were 2-3 times lower in the highest tertile than in the lowest tertile of intake. Furthermore, for current asthma, a borderline significant $(\mathrm{p}<0.10)$ difference in prevalence between the highest and lowest tertiles of intake of citrus fruits and vegetables was observed (table 2).

Linear associations of intake of citrus fruits, vegetables, whole grain products and fish with asthma end points are shown in table 3. Food intake was entered as a continuous variable into the logistic model and ORs are presented for the difference in intake (in g/day) between the highest and lowest tertile of intake (for mean food intake per tertile, see table 2). In the univariate analyses, fish intake showed an inverse linear association with both end points. A trend towards an inverse linear association $(\mathrm{p}<0.10)$ was observed for intake of citrus fruits with current asthma and for intake of whole grain products with both end points. After adjustment for potential confounders, fish intake remained inversely associated with both current wheeze and current asthma, and a trend towards an inverse association with both end points could still be observed for whole grain intake (table 3). Smoothing techniques showed that the curve of the association with fish intake was fairly linear for both end points, while the strongest decline in the prevalence of both end points was observed at whole grain intakes higher than $150 \mathrm{~g} /$ day.

When the intakes of whole grain products and of fish were studied simultaneously in a single model, the associations of the foods with either end point were largely independent of each other (table 3). Associations of the two foods with current asthma remained essentially unchanged after additional adjustment for intake of citrus fruits and vegetables. To gain some insight into the combined effect of whole grain and fish intake, children with a high intake (that is, in the highest tertile) of both foods were compared with those with a low intake (that is, in the lowest tertile) of both foods (table 4). The crude prevalence of current wheeze was observed to be $19.4 \%$ in children with a low intake of both foods compared with $4.2 \%$ in children with a high intake of both foods. For current asthma the crude prevalences were 16.7 and $2.8 \%$, respectively. These results may be compared with those in table 2 to evaluate additivity (see Discussion). The association of the combined intake of whole grain products and fish with the two end points remained essentially unchanged after adjustment for potential confounders (table 4).

The analyses in table 3 were repeated for the end points atopic wheeze with BHR and atopic asthma with BHR (for definitions see Methods section). Similar results to those for current wheeze and current asthma were observed. The adjusted ORs for the independent association with atopic wheeze with BHR was 0.31 (95\% CI 0.10 to 0.95 ) for whole grain intake and 0.15 (95\% CI 0.03 to 0.63 ) for fish intake. For the independent association with atopic asthma with BHR, the resulting adjusted ORs were 0.28 (95\% CI 0.08 to 0.99 ) for whole grain intake and 0.12 (95\% CI 0.02 to 0.66 ) for fish intake.

\begin{tabular}{|c|c|c|c|c|}
\hline \multirow[b]{2}{*}{ Selected foods } & \multirow[b]{2}{*}{ Tertile ${ }^{*}$} & \multirow{2}{*}{$\begin{array}{l}\text { Mean intake per } \\
\text { tertile (g/day) }\end{array}$} & \multicolumn{2}{|l|}{ Prevalence (\%) of: } \\
\hline & & & Current wheeze & Current asthma \\
\hline \multirow[t]{3}{*}{ Fruits, total } & $\mathrm{Tl}$ & 79 & 8.5 & 6.0 \\
\hline & T2 & 148 & 9.5 & 8.0 \\
\hline & T3 & 287 & 8.5 & 5.5 \\
\hline \multirow{3}{*}{ Citrus fruits } & $\mathrm{Tl}$ & 13 & 9.5 & 10.1 \\
\hline & T2 & 34 & 8.5 & $4.0 \ddagger$ \\
\hline & T3 & 81 & 8.5 & $5.5 \dagger$ \\
\hline \multirow[t]{3}{*}{ Vegetables, total } & $\mathrm{Tl}$ & 53 & 9.0 & 8.0 \\
\hline & T2 & 88 & 10.0 & 8.0 \\
\hline & T3 & 140 & 7.5 & $3.5 \dagger$ \\
\hline \multirow{3}{*}{ Dairy products, total } & $\mathrm{Tl}$ & 347 & 8.0 & 6.5 \\
\hline & T2 & 611 & 8.5 & 6.0 \\
\hline & T3 & 973 & 10.1 & 7.0 \\
\hline \multirow[t]{3}{*}{ Dairy, other than low fat } & $\mathrm{Tl}$ & 206 & 10.1 & 7.5 \\
\hline & T2 & 458 & 7.5 & 7.5 \\
\hline & T3 & 746 & 9.0 & 4.5 \\
\hline \multirow{3}{*}{ Whole grain products, total } & $\mathrm{Tl}$ & 1 & 13.1 & 10.6 \\
\hline & T2 & 15 & $7.0 \ddagger$ & $4.5 \ddagger$ \\
\hline & T3 & 118 & $6.5 \ddagger$ & $4.5 \ddagger$ \\
\hline \multirow{3}{*}{ Fish, total } & $\mathrm{Tl}$ & 1 & 13.1 & 10.6 \\
\hline & T2 & 6 & 8.6 & 6.1 \\
\hline & T3 & 15 & $5.0 \S$ & $3.0 \S$ \\
\hline
\end{tabular}


Table 3 Linear association of intake of selected foods with current wheeze and current asthma in children (ISAAC-2)

\begin{tabular}{|c|c|c|}
\hline Selected foods & $\begin{array}{l}\text { Current wheeze } \\
\text { OR* }^{*}(95 \% \mathrm{CI})\end{array}$ & $\begin{array}{l}\text { Current asthma } \\
\mathrm{OR}^{*}(95 \% \mathrm{Cl})\end{array}$ \\
\hline \multicolumn{3}{|c|}{ Individual associations } \\
\hline \multicolumn{3}{|l|}{ Citrus fruits $†$} \\
\hline Crude & $0.88(0.52$ to 1.48$)$ & $0.61(0.35$ to 1.05$)$ \\
\hline Adjusted $\neq$ & $0.96(0.56$ to 1.66$)$ & 0.65 (0.37 to 1.14$)$ \\
\hline \multicolumn{3}{|l|}{ Vegetables, total } \\
\hline Crude & $1.15(0.66$ to 2.00$)$ & $0.67(0.32$ to 1.38$)$ \\
\hline Adjusted $\neq$ & $1.24(0.69$ to 2.23$)$ & $0.69(0.33$ to 1.45$)$ \\
\hline \multicolumn{3}{|c|}{ Whole grain products, totalt } \\
\hline Crude & $0.53(0.26$ to 1.08$)$ & $0.44(0.19$ to 1.00$)$ \\
\hline Adjusted $\neq$ & $0.51(0.24$ to 1.08$)$ & $0.43(0.18$ to 1.02$)$ \\
\hline \multicolumn{3}{|l|}{ Fish, total } \\
\hline Crude & $0.40(0.19$ to 0.84$)$ & $0.31(0.13$ to 0.78$)$ \\
\hline Adjusted $\neq$ & $0.42(0.20$ to 0.89$)$ & 0.32 (0.13 to 0.81$)$ \\
\hline \multicolumn{3}{|c|}{ Independent§ associations (adjusted‡) } \\
\hline Whole grainst & $0.55(0.26$ to 1.15$)$ & $0.46(0.19$ to 1.10$)$ \\
\hline Fish & $0.44(0.21$ to 0.93$)$ & 0.34 (0.13 to 0.85 ) \\
\hline \multicolumn{3}{|c|}{$\begin{array}{l}\text { *Regression coefficient estimated modelling food intake as a continuous variable ( } \mathrm{g} / \text { day or natural log of } \mathrm{g} / \text { day) } \\
\text { and OR presented for the difference in mean intake (in } \mathrm{g} / \text { day) between the highest and lowest tertiles of intake (fo } \\
\text { mean food intake per tertile see table } 2 \text { ). } \\
\text { †Dietary variable modelled as natural log of } \mathrm{g} / \text { day. } \\
\text { †Adjusted for educational level of the mother, foreign descent, and total energy intake. } \\
\text { §The variables representing whole grain intake and fish intake were entered simultaneously in a single logistic } \\
\text { model. }\end{array}$} \\
\hline
\end{tabular}

Finally, the independent associations of the two foods with atopy and with BHR were studied. The adjusted ORs for the association with atopy were 1.42 (95\% CI 0.89 to 2.24 ) for whole grains and 1.28 (95\% CI 0.89 to 1.83 ) for fish. For BHR the adjusted ORs were 1.48 (95\% CI 1.01 to 2.16 ) for whole grains and 0.87 (95\% CI 0.58 to 1.32 ) for fish. No association with atopy or BHR was observed for the other foods studied.

\section{DISCUSSION}

We observed an inverse association of the intake of whole grain products and of fish with current wheeze and current asthma in children. Intake of these foods was furthermore inversely associated with more complex asthma end points based on both questionnaire and clinical data (atopic wheeze with BHR and atopic asthma with BHR). The associations of whole grain and fish intake with these asthma end points were largely independent of each other, and indications for additivity were observed. No clear associations with asthma were observed for intake of (citrus) fruits, vegetables, or dairy products.

A number of epidemiological studies have been published on fish intake and asthma in children with conflicting

Table 4 Combined intake of whole grain products and fish in relation to current wheeze and current asthma in children (ISAAC-2)

\begin{tabular}{|c|c|c|}
\hline \multirow[b]{2}{*}{ End points studied } & \multicolumn{2}{|c|}{ Whole grain products and fish } \\
\hline & $\begin{array}{l}\text { Intake of both } \\
\text { foods in lowest } \\
\text { tertile } \\
(\mathbf{n}=72)\end{array}$ & $\begin{array}{l}\text { Intake of both } \\
\text { foods in highest } \\
\text { tertile }(n=71)\end{array}$ \\
\hline \multicolumn{3}{|l|}{ Current wheeze } \\
\hline Crude prevalence (\%) & 19.4 & $4.2^{*}$ \\
\hline Adjusted† prevalence (\%) & 18.9 & $4.8^{*}$ \\
\hline \multicolumn{3}{|c|}{ Current asthma } \\
\hline Crude prevalence (\%) & 16.7 & $2.8^{*}$ \\
\hline Adjusted† prevalence (\%) & 16.1 & $3.4^{*}$ \\
\hline \multicolumn{3}{|c|}{$\begin{array}{l}\text { * } p<0.05 \\
\text { †Adjusted for educational level of the mother, foreign descent, and total } \\
\text { energy intake. }\end{array}$} \\
\hline
\end{tabular}

results. Some studies have reported potential protective effects of fish for asthma symptoms, ${ }^{69}$ BHR alone ${ }^{15}$ or in combination with wheeze, ${ }^{14}$ and atopic disease. ${ }^{13}{ }^{16}$ However, in other studies no association was observed with asthma symptoms, ${ }^{10} 12$ recent asthma, ${ }^{12}$ lung function, ${ }^{8}$ atopy, ${ }^{11}$ or atopic disease. ${ }^{13}$ In one study, ever asthma confirmed by a physician was observed to be positively associated with fish intake. ${ }^{29}$ Also, within studies, seemingly conflicting results have been reported. Hodge et $a l,{ }^{14}$ for instance, observed a potential protective effect for oily fresh fish but not for nonoily fresh fish or for canned or processed fish.

Fish oils-or, more specifically, the n-3 polyunsaturated fatty acids eicosapentaenoic acid and docosahexaenoic acidare thought to have anti-inflammatory effects through their effect on arachidonic acid metabolism. ${ }^{2}$ Despite the plausible biological mechanism, an important concern is the low level of fish intake at which potential effects are observed. For instance, the high fish intake group consumed any (versus no) fish in the study by Hodge et al, ${ }^{14}$ and consumed fish more than once a week in the study by Peat $e t$ al $^{15}$ and more than once a month in the study by Antova et al. ${ }^{6}$ In our study, children in the highest tertile of fish intake consumed on average $15 \mathrm{~g}$ of fish daily. It has been questioned whether fish intake at this level can have a substantial physiological effect on inflammatory processes in the airways. ${ }^{30}$ In placebo controlled trials in asthma patients, supplementation with high doses of fish oils has been observed to have inhibitory effects on neutrophils. ${ }^{31}{ }^{32}$ However, in asthma other inflammatory cells such as eosinophils and mast cells seem to play a more predominant role. ${ }^{30}$ The fact is that, in the abovementioned trials, no clinical benefit of fish oil supplementation was observed.

Our findings regarding whole grain intake are in line with those reported by Wijga et $\mathrm{al}^{12}$ and suggest a protective effect against asthma in children. COPD in adults has also been observed to be inversely associated with whole grain intake. ${ }^{33}$ Whole grains are rich in antioxidant compounds (including vitamin E, phenolic acid and phytic acid), ${ }^{4}{ }^{5}$ and a high intake of whole grain products may therefore help to protect the airways against oxidant damage.

Inverse associations with asthma related outcomes in children have been reported for (citrus) fruits $^{67}$ and 
vegetables, ${ }^{8-11}$ foods also rich in antioxidant compounds. No association between vegetable intake and asthma related outcomes in children was found in one study. ${ }^{12}$ Most published epidemiological studies found no association between fruit intake and asthma. ${ }^{8}{ }^{10-12}{ }^{34}$ In our study, no association was found between total fruit intake and the studied outcomes (including atopy and BHR individually). The associations with studied outcomes were also not statistically significant for intake of citrus fruits or vegetables. The results regarding current asthma, however, do suggest a potential inverse association with both foods.

In contrast to a number of epidemiological studies, ${ }^{10} 121819$ we did not observe an inverse association between the intake of dairy products (total or other than low fat products) and asthma. The intake of these foods can be seen as a marker for intake of saturated fatty acids. Data on fatty acid intake were not available, and different types of fatty acids (n-6, n-3, and saturated fatty acids) could therefore not be studied in relation to asthma.

The associations of intake of whole grain products and fish with asthma were largely independent of each other. The fact that the observed difference in the prevalence of current wheeze and of current asthma was smaller in children with a high versus low intake of either food (table 2) than in children with a high versus low intake of both foods (table 4) suggests that the effects of the two foods may be (partly) additive. Our attempts to study this aspect were limited by the fact that the size of the study population did not allow for more detailed stratification.

When intake of the selected foods was studied in relation to atopy and to BHR, a positive association between whole grain intake and BHR was observed. Furthermore, atopy tended to be positively associated with the intake of both whole grain products and fish. To our knowledge, similar findings have not been reported previously. It needs to be established whether these are chance findings or whether they can be confirmed in other epidemiological studies.

A high intake of whole grain products and of fish may reflect a more healthy diet and even a more healthy lifestyle in general. However, we observed no clear confounding effects of other health related lifestyle factors on the observed associations of whole grain and fish intake with asthma outcomes. Adjustment for intake of fruits or vegetables, generally considered to be healthy, did not cause a significant change in these associations (results not shown). The same was observed when adjusting for an indicator of socioeconomic status of the family (educational level of the mother), which is generally considered to be associated with a healthy lifestyle. Furthermore, in preliminary analyses, no strong confounding effects were observed for smoking by the mother during pregnancy, being breastfed, passive smoking, or body mass index. However, since both healthy lifestyle and socioeconomic status are complex concepts, residual confounding cannot be excluded.

A validated questionnaire for assessing dietary intake in children is and was, to the best of our knowledge, not available in the Netherlands. We used the EPIC FFQ, validated in Dutch adults, ${ }^{21}$ and asked parents to complete this questionnaire with regard to their child's diet. This allowed us to study the habitual intake, being the exposure of interest in relation to disease, of a broad range of dietary factors. The answer categories of all questions on relative or absolute frequencies of food consumption and portion sizes encompassed the whole range of intake. Potentially relatively small (frequencies of) intakes in children could therefore be entered without problem. FFQs are widely used in epidemiological research and have been shown to be reasonably valid for ranking individuals according to food group, as was also observed for the EPIC FFQ in adults. ${ }^{21}$ However, since the EPIC FFQ was not validated for use in children, our results have to be interpreted with additional caution. In most studies on diet and asthma in children published to date, non-validated questions and questionnaires have been used to assess dietary intake. The development and validation of dietary questionnaires for children seems to be an important area for future research.

In conclusion, our findings suggest that a high intake of whole grain products and fish may have a protective effect against asthma in children. Based on our study, causal inferences cannot be made. Prospective studies in which dietary factors (fish and whole grain products, but preferably also fruits and vegetables) and asthma are monitored and trials into the effect of dietary modifications on, for example, airway inflammation are needed to clarify this issue.

\section{Authors' affiliations}

C Tabak, A H Wijga, H A Smit, Center for Prevention and Health Services Research, National Institute of Public Health and the Environment, Bilthoven, The Netherlands

G de Meer, N A H Janssen, B Brunekreef, Environmental and Occupational Health, Institute for Risk Assessment Sciences, Utrecht University, Utrecht, The Netherlands

G de Meer, Department of Health Sciences, University Medical Center Groningen, Groningen, The Netherlands

This study was financially supported by the Ministry of the Environment of The Netherlands and by the Netherlands Asthma Foundation.

Competing interests: none declared.

\section{REFERENCES}

1 Seaton A, Godden DJ, Brown K. Increase in asthma: a more toxic environment or a more susceptible population? Thorax 1994:49:171-4.

2 Simopoulos AP. Essential fatty acids in health and chronic disease. Am J Clin Nutr 1999;70(Suppl):P560-9.

3 Greene LS. Asthma, oxidant stress, and diet. Nutrition 1999;15:899-907.

4 Thompson LU. Antioxidants and hormone-mediated health benefits of whole grains. Crit Rev Food Sci Nutr 1994;34:473-97.

5 Slavin J, Jacobs D, Marquart L. Whole-grain consumption and chronic disease: protective mechanisms. Nutr Cancer 1997;27:14-21.

6 Antova T, Pattenden S, Nikiforov B, et al. Nutrition and respiratory health in children in six Central and Eastern European countries. Thorax 2003:58:231-6.

7 Forastiere F, Pistelli R, Sestini P, et al. Consumption of fresh fruit rich in vitamin $\mathrm{C}$ and wheezing symptoms in children. Thorax 2000;55:283-8.

8 Cook DG, Carey IM, Whincup PH, et al. Effect of fresh fruit consumption on lung function and wheeze in children. Thorax 1997:52:628-33.

9 Ellwood P, Asher MI, Björkstén B, et al. Diet and asthma, allergic rhinoconjuctivitis and atopic eczema symptom prevalence: an ecological analysis of the International Study of Asthma and Allergies in Childhood (ISAAC) data. Eur Respir J 2001; 17:436-43.

10 Hijazi N, Abalkhail B, Seaton A. Diet and childhood asthma in a society in transition: a study in urban and rural Saudi Arabia. Thorax 2000;55:775-9.

11 Remes ST, livanainen K, Koskela H, et al. Which factors explain the lower prevalence of atopy amongst farmers' children. Clin Exp Allergy 2003:33:427-34.

12 Wijga AH, Smit HA, Kerkhof $M$, et al. Association of consumption of products containing milk fat with reduced asthma risk in pre-school children: the PIAMA birth cohort study. Thorax 2003;58:567-72.

13 Dunder T, Kuikka L, Turtinen J, et al. Diet, serum fatty acids, and atopic diseases in childhood. Allergy 2001;56:425-8.

14 Hodge L, Salome CM, Peat JK, et al. Consumption of oily fish and childhood asthma risk. Med J Aust 1996;164:137-40.

15 Peat JK, Salome CM, Woolcock AJ. Factors associated with bronchial hyperresponsiveness in Australian adults and children. Eur Respir J 1992:5:921-9.

16 Nafstad P, Nystad W, Magnus $P$, et al. Asthma and allergic rhinitis at 4 years of age in relation to fish consumption in infancy. $J$ Asthma 2003;40:343-8.

17 Bolte G, Frye C, Hoelscher B, et al. Margarine consumption and allergy in children. Am J Respir Crit Care Med 2001;163:277-9.

18 Riedler J, Braun-Fahrländer C, Eder W, et al. Exposure to farming in early life and development of asthma and allergy: a cross-sectional survey. Lancet 2001;358:1129-33.

19 von Ehrenstein OS, von Mutius E, Illi S, et al. Reduced risk of hay fever and asthma among children of farmers. Clin Exp Allergy 2000;30:187-93.

20 Janssen NAH, Brunekreef $B$, van Vliet $P$, et al. The relationship between air pollution from heavy traffic and allergic sensitization, bronchial hyperresponsiveness, and respiratory symptoms in Dutch schoolchildren Environ Health Perspect 2003;111:1512-8.

21 Ocké MC, Bueno de Mesquita HB, Goddijn HE, et al. The Dutch EPIC food frequency questionnaire I. Description of the questionnaire, and relative validity and reproducibility for food groups. Int J Epidemiol 1997;26(Suppl):P37-48. 
22 Stichting NEVO. Nederlands voedingsstoffenbestand. The Hague: Voorlichtingsbureau voor de Voeding, 1996.

23 Asher MI, Keil U, Anderson HR, et al. International study of asthma and allergies in childhood (ISAAC): rationale and methods. Eur Respir J 1995;8:483-91.

24 Riedler J, Reade T, Dalton $M$, et al. Hypertonic saline challenge in an epidemiologic survey of asthma in children. Am J Respir Crit Care Med 1994; 150:1632-9.

25 Riedler J, Reade T, Robertson CF. Repeatability of response to hypertonic saline aerosol in children with mild to severe asthma. Pediatr Pulmonol 1994;18:330-6.

26 European Respiratory Society. Standardized lung function testing. Official statement of the European Respiratory Society. Eur Respir J 1993;6(Suppl): 1-100

27 ISAAC. Phase II modules of the International Study of Asthma and Allergies in Childhood. Münster, Germany: University of Münster, 1998.

28 EAACI. Skin tests used in type I allergy testing (position paper). Subcommittee on Skin Tests of the European Academy of Allergology and Clinical Immunology. Allergy 1989;44(Suppl 10): 1-59.
29 Takemura $Y$, Sakurai $Y$, Honjo S, et al. The relationship between fish intake and the prevalence of asthma: the Tokorozawa Childhood Asthma and Pollinosis study. Prev Med 2002;34:221-5.

30 Thien FCK, Woods RK, Walters EH. Oily fish and asthma-a fishy story? Further studies are required before claims can be made of a beneficial effect of oily fish consumption on asthma. Med J Aust 1996;164:135-6.

31 Arm JP, Horton CE, Mencia-Huerta J-M, et al. Effect of dietary supplementation with fish oil lipids on mild asthma. Thorax 1988:43:84-92.

32 Arm JP, Horton CE, Spur BW, et al. The effects of dietary supplementation with fish oil lipids on the airway response to inhaled allergen in bronchial asthma. Am Rev Respir Dis 1989;139:1395-400.

33 Tabak C, Smit HA, Heederik D, et al. Diet and chronic obstructive pulmonary disease: independent beneficial effects of fruits, whole grains, and alcohol (the MORGEN study). Clin Exp Allergy 2001;31:747-55.

34 Huang S-L, Pan W-H. Dietary fats and asthma in teenagers: analyses of the first Nutrition and Health Survey in Taiwan (NAHSIT). Clin Exp Allergy $2001 ; 31: 1875-80$

\section{LUNG ALERT}

\section{Deficient innate immunity and susceptibility of asthmatics to infection}

$\Delta$ Contoli M, Message SD, Laza-Stanca V, et al. Role of deficient type III interferon- $\lambda$ production in asthma exacerbations. Nat Med 2006;12:1023-6

$\mathrm{R}$ hinoviruses are a major cause of asthma exacerbations. Asthmatics may have increased susceptibility to rhinoviral (and bacterial) infections but the mechanisms involved are not known. This study aimed to identify a possible mechanism of susceptibility involving induction of type III interferon, interferon $\lambda$.

Primary bronchial epithelial cells from asthmatic and normal subjects were infected with rhinovirus and levels of viral RNA expression were measured. Viral replication was increased in cells from asthmatic subjects compared with normal subjects. Furthermore, both the expression of mRNA encoding type III interferons as well as the level of interferon $\lambda$ protein was decreased in asthmatics compared with normal volunteers. Levels of mRNA encoding the type III interferon and levels of interferon $\lambda$ protein were inversely related to levels of viral RNA. The production of interferon $\lambda$ by bronchoalveolar lavage (BAL) cells (mainly macrophages) from asthmatic and normal subjects infected with rhinovirus was also measured. Production of interferon $\lambda$ was again lower in cells from asthmatics than in cells from normal subjects.

After the ex vivo studies the same volunteers were inoculated with rhinovirus 2 . Significant inverse correlations were observed between the ex vivo production of interferon $\lambda$ and severity of symptoms, BAL fluid virus load, and airway inflammation. The increased risk of bacterial infection was assessed by incubating BAL cells from the same subjects with lipopolysaccharide and measuring type III interferon production. Significant induction of interferon $\lambda$ was noted, but this was impaired in asthmatic subjects.

This study highlights a possible mechanism in the pathogenesis of asthma exacerbations involving type III interferon.

A Adams

FY2 Doctor, Royal Free Hospital, London, UK; ashokadams@hotmail.com 